\title{
Simple drawing and pattern completion techniques for studying visualization and long-term visual knowledge
}

\author{
D. F. M. CHRISTIE and W. A. PHILLIPS \\ Stirling University, Stirling FK9 4LA, Scotland
}

\begin{abstract}
Simple and efficient drawing and completion tasks for studying visual memory are developed. In Experiment 1 subjects reproduced a series of matrix patterns by filling empty matrices. The serial position function was flat, except that accuracy was much higher for final patterns. In Experiment 2 this recency effect was removed by an interpolated pattern classification task. Experiments 3 and 4 examined the effect of counting backward during intervals of from 3 to 15 sec on the recall of single patterns. Drawings were much less accurate after filled intervals but the duration of the interval had no effect. Experiment 5 tested retention of series of patterns using a completion task. On immediate test the serial position function was the same as in Experiments 1 and 2. On a final test accuracy was unchanged except for final items, which then showed a small negative recency effect. It is argued that performance is so similar in the drawing, completion, and previously reported recognition tasks because in all it is based upon the use of general-purpose knowledge accessible to voluntary processing. Visualization in these tasks is analogous to but different from verbal STM. One main difference is that there is no sign of temporary storage of visualized information after attention has turned to other things.
\end{abstract}

Many investigations of both normal and pathological function suggest that human visual cognition involves components analogous to the short-term memory (STM) and long-term memory (LTM) components of verbal cognition (e.g., Kroll, 1975; Warrington \& Weiskrantz, 1973). One way of investigating this functional specialization is by studying memory for novel visual configurations. Memory for a series of such patterns has recency and long-term components that are even more distinct than are the analogous components in verbal memory (Phillips \& Christie, 1977a, 1977b). These experiments used single-item probe or reverse serial order testing techniques and found a large recency effect for the final item of a series but a completely flat serial position function otherwise. Mental arithmetic during the retention interval entirely removed the advantage to the final item but did not affect performance on the other items, producing a completely flat serial position function. Conversely, presentation rate did not affect performance on the final items but did affect performance on the other items (Phillips \& Christie, 1977a). The interpretation offered was that performance on the final item depended upon active visualization of that item throughout the interval between presentation and test, whereas performance on all other items depended upon whatever long-term knowledge had been

This work was supported by a grant from the United Kingdom Science Research Council. Reprint requests should be sent to W. A. Phillips, Psychology Department, Stirling University, Stirling FK9 4LA, Scotland. D. F. M. Christie is now at the Department of Psychology, University of Aberdeen, Scotland. acquired of them during presentation. Further support for this interpretation comes from as yet unpublished work by Steve Avons at the University of Stirling that shows that if initial presentation is brief, then presentation time affects both components, but in very different ways.

Thus the results obtained by Phillips and Christie (1977a) were strikingly similar to those obtained with verbal materials in showing distinct recency and longterm components. However, there were also clear differences between these results and those obtained with verbal materials: (1) The recency component extended over only one item rather than over four or five. (2) There was no sign of primacy effects. (3) As in the experiment by Shaffer and Shiffrin (1972), the long-term component was unaffected by the duration of the blank interval between items in a series. (4) The recency component was reduced but not removed by a 10-sec unfilled retention interval. (5) Recency occurred only under STM conditions; under LTM conditions, the serial position function was flat.

The experiments of Phillips and Christie (1977a) were therefore interpreted as strong support for the view that visual cognition had components analogous to but different from the STM and LTM components of verbal cognition. In all those experiments, however, the task was recognition. At test the subject was shown a pattern that was either identical to a previously presented pattern or slightly different. His task was to decide whether it was identical or not. Therefore, the conclusions drawn from those experiments may apply only 
to recognition memory, and perhaps only to this particular kind of recognition test. The aim of the present experiments is to determine whether the pattern of functional interactions found for recognition generalize to quite different tasks using the same visual material. In other words, we are asking whether performance in the recognition experiments depends upon knowledge especially designed to enable patterns to be recognized, or upon knowledge that can be put to any of a wide variety of uses.

The two tasks studied here are drawing and pattern completion. The patterns used were 4 by 4 matrices of square cells, with about half filled at random: Drawing involved filling cells of empty matrices by simple strokes of a broad felt-tipped pen. Pattern completion involved filling one extra cell of a matrix pattern to make it into a pattern previously presented. There were two main empirical questions: (1) Do drawing and completion tasks show clear STM and LTM components and, if so, are these components similar to those in visual recognition tasks? (2) Do drawing and completion differ from verbal performance in ways similar to that in which recognition does?

Drawing differs from recognition in so many ways that quite different results seem likely. Reproduction of matrix patterns has occasionally been used to study short-term visual retention (e.g., Adamowicz \& Hudson, 1978; Schnore \& Partington, 1967; Yuille \& Ternes, 1975), and a recent study (Phillips, Hobbs, \& Pratt, 1978) suggests that some kind of visual STM is used when copying or drawing from life. However, no direct attempt seems to have been made to show distinct STM and LTM components in drawing tasks. The pattern completion task, although simple, has been little used.

The tasks used to test memory are important in relation to the contrast between visual and verbal performance. Nearly all the verbal experiments use recall tasks, and nearly all the visual experiments use recognition tasks. It is possible that the differences between visual and verbal performance arise from this difference in task. To the extent that this is so, drawing should produce results more similar to the verbal results than does recognition. Cohen and Granstrom (1970) have reported evidence that visual recall tasks are more dependent upon verbal mediation than are visual recognition tasks. This again suggests that drawing will differ from recognition and be more like verbal performance. Even so, it seems unlikely that all the differences between visual and verbal performance are due to differences in the tasks used because studies of verbal STM using probe recognition tasks (Raser, 1972; Shulman, 1970) obtain rather conventional verbal serial position curves.

The present experiments also have a methodological purpose. The great emphasis within recent visual memory research on recognition tasks of various kinds is both misleading and inefficient. It is misleading because it suggests that visual knowledge is in general particularly designed for recognition. As a consequence of this emphasis, there is neglect of the way in which visual knowledge guides action. Recognition methods are inefficient because they produce little information per trial, and require many trials to give significant results. Drawing was commonly used in the 1940s and 1950s to test the Gestalt theory of the autonomous change of the memory trace (see Riley, 1962). That theory proved sterile, however, and was abandoned. Interest in drawing has largely been abandoned with it. Simplicity of execution may be necessary if drawing is to reflect visualization, because visualization seems to be a demanding process and is easily disrupted (Phillips \& Christie, $1977 \mathrm{~b}$ ). If the reproductive technique demands attention, any new information being maintained by visualization may be lost. The tasks used were therefore carefully designed so that they are easily understood and easily executed. They were also designed such that the materials required are simple, cheap, and portable.

\section{DRAWING: SERIAL POSITION EFFECTS}

\section{Experiment 1}

\section{Method}

Subjects. The subjects were eight undergraduates who volunteered to satisfy a requirement of an introductory psychology course.

Materials. The stimuli were 4 by 4 square matrices containing eight black and eight white cells. A total of 60 such patterns were generated randomly and drawn with a cell size of $6 \mathrm{~mm}$ on plain white stimulus cards for presentation by means of an automatically timed shutter mechanism. Viewing distance was approximately $50 \mathrm{~cm}$. The stimulus cards were divided into sets of 4,10 sets to be used in the test trials and 5 sets for practice.

Procedure. A trial consisted of the presentation of a series of four patterns followed by a period of "free recall," during which subjects attempted to reproduce the configuration of black cells in each matrix pattern. This was done by filling the corresponding cells in blank matrices on the response sheets provided, using strokes of a broad fiber-tipped marking pen. Presentation rate was one pattem per $3.5 \mathrm{sec}$ ( $2 \mathrm{sec}$ on and $1.5 \mathrm{sec}$ off). As a recall cue, a blank stimulus card was displayed $1.5 \mathrm{sec}$ after the offset of the final pattern of each series. Subjects were allowed to draw the four patterns in any order without time limit, but after completing the four reproductions, subjects were required to arrange them in an order, from left to right, as closely matching the serial order of presentation as possible. At the end of this recall phase, the four stimulus cards we re laid out in front of the subject in the order of presentation. Any obvious discrepancy noticed by the subject of the experimenter in the order in which the reproductions were arranged was recorded and then rectified. Each reproduction was later scored against the pattern from the series of four presented on that particular trial that it most closely resembled, with the restriction that the same pattern was never used as standard for more than one reproduction. In any cases of ambiguity, the position in which the subject had placed the reproduction after recall was used as a guide to which pattern should be used as standard.

The presentation order of the patterns within the sets of four 
was balanced across subjects so that each pattern appeared twice in each serial position over the experiment as a whole. Subjects were tested individually in a session lasting about $1 \mathrm{~h}$ that consisted of 10 test trials preceded by 5 practice trials.

\section{Results}

Each reproduction received a score of between 0 and 16 according to the number of cells correctly filled in the 4 by 4 matrix. Thus over the 10 trials, a maximum total score of 160 for each serial position was possible for each subject. The overall percentage of cells correct is shown in Figure 1a as a function of serial presentation position. A one-way analysis of variance with repeated measures of the number of cells correct indicated a highly significant effect of serial position $[F(3,21)=$ $12.74, p<.001]$ due entirely to the superiority of the final position over each other serial position, as confirmed by Newman-Keuls analysis. There were no significant differences among Positions 1.3 $(\alpha=.05)$.

The percentage of cells that would be filled correctly by chance if a subject filled eight cells at random on each trial could be expected to average $50 \%$, since the patterns have equal numbers of white and black cells. The level of performance at Positions 1-3 of approximately $65 \%$ is well above this level. A more stringent measure of performance is the number of patterns reproduced completely correctly (i.e., receiving a score of 16). The mean scores for Serial Positions 1-4 on this measure were $1.25,1.375,1.0$, and 3.2 , respectively, out of a possible 10 . It is clear from both measures that the final pattern in a series of four is recalled more accurately than the other three, which are recalled with about equal accuracy. This serial position function is strikingly similar to that found with a recognition task (Phillips \& Christie, 1977a), which was interpreted as evidence that only a single pattern can be visualized. Many subjects, on debriefing, reported having been able to visualize accurately only the final pattern at recall. Although it was stressed that pattems could be drawn in any order, subjects mostly chose to start with the final pattern, which was still "in mind" when they came to draw.

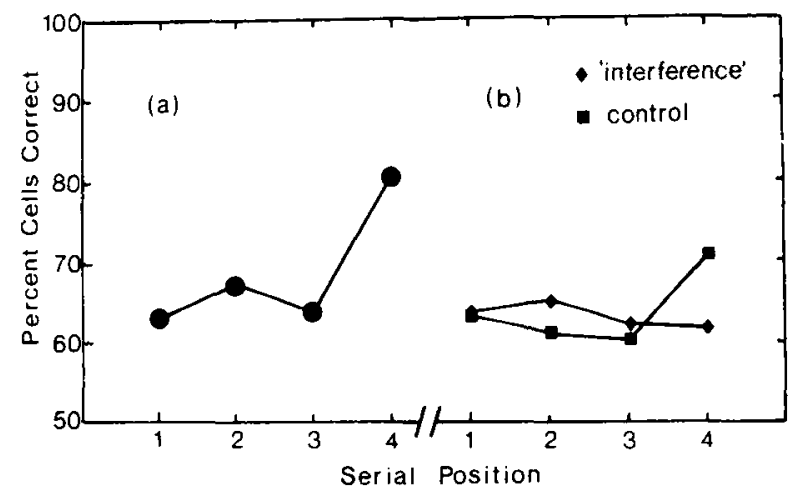

Figure 1. Mean percentage of cells correctly filled: (a) in Experiment 1 as a function of serial position, (b) in Experiment 2 as a function of serial position and intervening activity.

\section{Experiment 2}

It has become clear in the last few years that not all recency effects are removed by intervening activity or long intervals (e.g., Bjork \& Whitten, 1974; Tzeng, 1973; Baddeley \& Hitch, Note 1). Evidence for STM, therefore, requires recency effects that are removed by intervening activity. In our studies of visual recognition memory, we found that all recency effects were entirely removed by just a few seconds of intervening activity. Experiment 2 asks whether this also applies to recall.

\section{Method}

Subjects. Sixteen new subjects were enlisted from the same subject pool as that used in Experiment 1. Subjects were randomly assigned to one of two groups of eight.

Materials and Procedure. The same sets of patterns were used as in Experiment 1 and the same procedure was followed, except for the manipulation of the type of activity required during the interval between presentation and recall. At the beginning of the session, the experimental group of subjects was trained to discriminate between two similar matrix patterns (of the same type as the memory material) by associating the symbol " $\sqrt{ }$ " (check) with one pattern and " $X$ " (cross) with the other. On each trial, one of these two patterns was presented for $2 \mathrm{sec}, 1.5 \mathrm{sec}$ after the offset of the fourth pattern. The two patterns occurred equally often throughout the experiment in an order that was predetermined but apparently random across trials. Before attempting to reproduce the four patterns, subjects in this group were required to place the appropriate symbol in a space provided on the response sheet, depending on which of the two possible patterns had been displayed on that trial.

For the control group of subjects, a redundant "checkerboard" pattern was presented after the fourth pattern in each series. These subjects were instructed to wait until this pattern had been displayed, put a check or a cross in the space provided on the response sheet, and then attempt to draw the four patterns. Half of this control group always put a check before starting to draw, the other half always put a cross.

Each of the eight subjects in each group received 5 practice and 10 test trials individually in a session lasting about $1 \mathrm{~h}$.

\section{Results}

The mean percentage of cells correctly recalled is shown in Figure $1 \mathrm{~b}$ as a function of serial position and type of interpolated activity. An analysis of variance on the number of cells correct with type of interpolated activity as a between-subjects variable and serial position as a within-subjects variable indicated no overall effect of type of activity $(F<1)$ and no significant effect of serial position $[F(3,42)=2.06, p>.1]$. However, the interaction between these two factors was significant $[F(3,42)=4.45, p<.01]$. From inspection of Figure $1 b$, this appears to be largely due to the difference between groups at Position 4. In a separate $t$ test, this difference in performance on the fourth pattern proved significant $[t(8)=2.39, p<.05]$.

A one-way analysis of variance of the control scores alone yielded a significant effect of serial position $[F(3,21)=7.03, p<.01]$, due to the advantage of the final serial position. However, a similar analysis of scores for the "interference" group showed no significant 
effects of serial position. The control group produced basically the same shape of serial position curve as was found in Experiment 1, but inspection of Figure 1 indicates that the size of the recency effect in absolute terms was reduced. To test whether this reduction was significant, the scores for Position 4 of the eight subjects in the control group were compared with those of the eight subjects in Experiment 1 by means of a MannWhitney test. This comparison showed a significant difference $\left[\mathrm{U}^{1}(8,8)=8.5, \mathrm{p}<.05\right]$. This suggests that the appearance of a redundant pattern $1.5 \mathrm{sec}$ after the offset of the last pattern and a requirement to make a simple response prior to recall cause some interference with performance on the final item. However, the recency effect is completely absent for the group performing the pattern classification task. The interpolated activity reduces performance only for the final pattern in a string.

\section{Discussion}

The primary outcome of the first two experiments is that the serial position function for drawing and the way it is affected by interference during the retention interval are the same as that found earlier for recognition. The serial position function for drawing also contrasts with that typical of verbal performance in the same way as does that for recognition: The recency effect was confined to one item; it was entirely removed by interference; there was no evidence of primacy. List length was only four items, but recognition experiments have shown that increasing list length from two to eight items has no effect upon either the absolute level or the shape of the serial position function for these patterns (Phillips \& Christie, 1977a). Verbal memory experiments must use longer lists because if only four were used, little would be seen beyond the recency component.

The present serial position functions differ from those obtained with verbal recall of pictures that normally show the same primacy and recency effects as for verbal material (e.g., Tabachnick \& Brotsky, 1976). This is probably because verbal recall leads to the use of verbal representations.

Drawing is not only very similar to recognition in its functional dependence on serial position and interference, but it is also similar in terms of the absolute level of performance. Comparison of performance in Experiment 1 with that in the recognition experiment most similar in terms of pattern complexity, presentation rate, list length, and retention interval (Phillips \& Christie, 1977a, Experiment 6, Figure 6a) shows that, at each serial position, the probability that cells will be correctly filled when drawing is practically identical to the probability of knowing whether or not a test pattern has a single cell incorrectly filled. The percent cells correct in Experiment 1 above were $63.2 \%, 67.5 \%$, $63.8 \%$, and $80.2 \%$ for Positions $1-4$, respectively. Performance in the recognition experiment was $61.9 \%$, $65.2 \%, 60.6 \%$, and $78.4 \%$, respectively.

\section{DRAWING: THE BROWN-PETERSON PARADIGM}

The Brown-Peterson paradigm (Brown, 1958; Peterson \& Peterson, 1959) initiated contemporary research on verbal STM and remains central to the common conception of STM. The standard interference task of counting backward by threes is also used in our visual memory tasks because mental arithmetic has been shown to remove visualization in such tasks (Phillips \& Christie, 1977b).

\section{Experiment 3}

\section{Method}

Subjects. The subjects were 12 new undergraduate volunteers from the same subject pool as was used in the first two experiments.

Design and Procedure. Each trial consisted of the presentation of a single matrix pattern for $1.5 \mathrm{sec}$ followed by a retention interval of $3,7.5$, or $15 \mathrm{sec}$ either blank or filled with the distractor task of Peterson and Peterson (1959), at the end of which the subject received a cue to draw the pattern. On trials with filled intervals, the experimenter read a three-digit number on the offset of the stimulus pattern. Subjects were instructed to count backward by threes as rapidly as possible starting from this number until the recall cue was given. There were six conditions, resulting from the combination of the two factors: retention interval (3) by interference (2). Each subject performed a block of five trials in each condition, with the order of the blocks balanced across subjects by a Latin square design. Each of the 30 patterns used, drawn from the same set as used in the previous two experiments, appeared equally often in each condition across subjects. Testing was carried out individually in a session lasting about $1 \mathrm{~h}$ that included a block of 12 practice trials ( 2 per condition) in addition to the 30 test trials.

\section{Results}

The percentage of cells correctly reproduced is shown in Figure 2 as a function of retention interval and interpolated activity. The number of cells correct was subjected to a two-way analysis of variance with repeated measures. The effect of interpolated activity was highly significant $[F(1,11)=86.49, p<.001]$. There was no effect of length of retention interval and no significant interaction (both $\mathrm{Fs}<1$ ).

\section{Experiment 4}

In Experiment 3 the interval from the end of one trial to the beginning of the next was held constant. Therefore, there was a shorter time between the presentation of successive patterns when retention interval was short. It is possible that this may hide a reduction in performance with retention interval by increasing proactive interference in the blocks of trials with short retention intervals. Although possible, this seems un. likely, however, because proactive interference in visual memory is small and asymptotes after one trial (Meudell, 1977), and because over many years of work with this paradigm, we have seen no such effects. Nevertheless, this possibility is investigated in this experiment. 


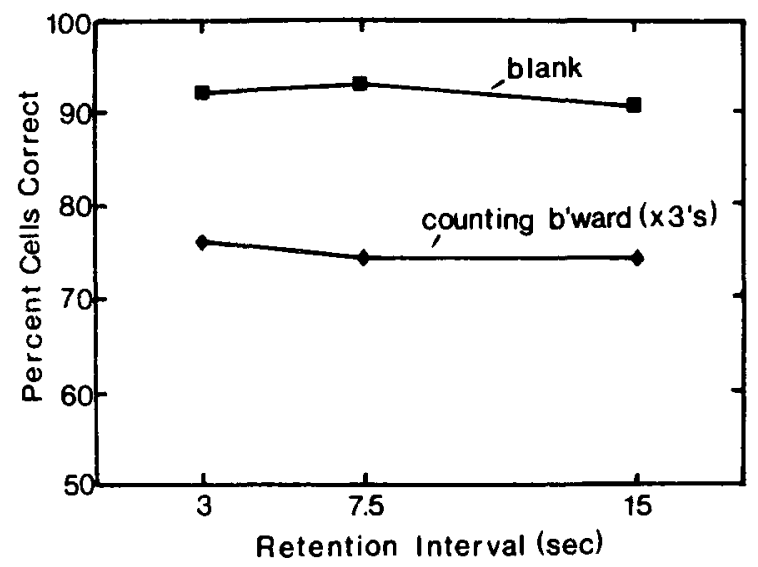

Figure 2. Mean percentage of cells correctly filled in Experiment 3 as a function of the duration of the retention interval and the intervening activity.

\section{Method}

Subjects. The subjects were eight postgraduate research workers in the Department of Psychology at the University of Stirling.

Design and Procedure. The basic difference from Experiment 3 was that the time between the beginning of successive trials was held constant at $40 \mathrm{sec}$ for all conditions. All else was the same except that only retention intervals of 3 and $15 \mathrm{sec}$ were studied. There were thus four conditions: retention in terval (2) by interference (2). Each subject performed a block of 10 trials in each condition, with the order of conditions balanced across subjects by a Latin square design.

\section{Results}

The percentage of cells correctly filled is shown in Figure 3 as a function of retention interval and interpolated activity. The number of cells correct was subjected to a two-way analysis of variance with repeated measures. The effect of interpolated activity is highly significant $[F(1,7)=33.79, \mathrm{p}<.001]$. Neither the effect of retention interval nor the interaction approaches significance (both $\mathrm{Fs}<1$ ).

Figure 3 also shows the percentage of patterns drawn completely correctly in each of the four conditions. An analysis of variance on the number of patterns completely correct shows a highly significant effect of interpolated activity $[F(1,7)=31.5, p<.001]$. No other effect approaches significance.

(a)

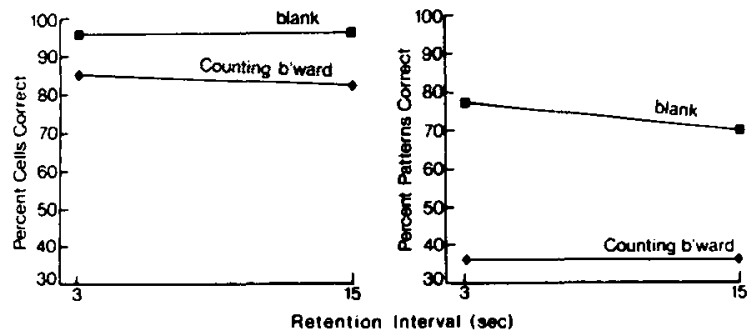

Figure 3. Performance in Experiment 4 as a function of the duration of the retention interval and the intervening activity: (a) the percentage of cells correctly filled, (b) the percentage of patterns drawn completely correctly.

\section{Discussion}

Experiments $1-4$ all show that the drawing task used here is simple and efficient. Performance was high in the unfilled conditions of Experiments 3 and 4 . Just a few seconds of counting backward, however, causes numerous errors. Together with the significant recency in Experiments 1 and 2, this is evidence that the act of drawing did not disrupt visualization.

The absence of any effect of retention interval was a surprise. Experiment 4 shows that this is a reliable result, and that it is not due to higher levels of proactive interference at the shorter retention intervals. The result is a surprise because verbal memory shows marked decay over filled retention intervals, and because many previous investigations of visual recognition memory for such patterns have shown clear decay over unfilled intervals (e.g., Phillips \& Baddeley, 1971). Thus recall seems to deteriorate less rapidly than recognition in visual STM. This may appear to conflict with the findings of Rock and Engelstein (1959), who found much more rapid decay for recall than for recognition in visual memory. The apparent contradiction can be resolved by noting that their results relate to visual LTM, whereas ours relate to visual STM.

Verbal STM does not decay over blank intervals that allow rehearsal. Thus, in this respect, drawing is more like verbal STM than is recognition. However, a new difference now appears. Drawing accuracy does not decay over filled intervals, whereas verbal STM does. Counting backward does interfere greatly with drawing accuracy but, instead of initiating gradual decay of performance, it seems to bring performance down to asymptote very rapidly.

\section{PATTERN COMPLETION: SERIAL POSITION EFFECTS}

The drawing task has some weaknesses. One is that although simple for most subjects, there may be some classes of subjects, such as very young children or neuropsychological patients, for whom it could present difficulties. Another weakness is that as list length increases, it will become less clear which pattem in the presentation series a reproduction should be paired with in order to score it. Finally, as with all free recall tasks, it is difficult to separate learning of the pattern itself from the acquisition of retrieval cues to it. The completion task reduces these difficulties. In this task the subject simply places a small plastic square in one of the empty cells of a matrix pattern so as to make it into a pattern previously presented.

\section{Experiment 5}

\section{Method}

Subjects. The subjects were 16 students and members of staff of the psychology department.

Materials. The stimuli were photographs of matrix patterns 
constructed by placing black and white plastic squares in a 4 by 4 grid. Pairs of patterns, one containing seven and the other six white cells, were constructed in this way, and prints were prepared in which each cell measured $2.5 \mathrm{~cm}^{2}$. The pairs of patterns were identical except that one member, the test pattern, had one less white cell than the other. Responses were made by placing a $2.5-\mathrm{cm}$ white plastic square on top of each test pattern. Twenty different patterns were used, and these were divided into sets of four for serial presentation.

Procedure. On each trial a series of four patterns was presented. These were laid out in a row one at a time on a table in front of the subject. Each pattern was presented for $2 \mathrm{sec}$, then a thick piece of card was placed over it, obscuring the pattern, before the next was presented. The presentation and interstimulus intervals of $2 \mathrm{sec}$ were timed by the experimenter using a battery-operated metronome with earpiece set to tick at a $1-\sec$ rate. After a retention interval of $4 \mathrm{sec}$ following the fourth pattern, the four "incomplete" test patterns were presented in reverse serial order, fourth pattern tested first. (For a discussion of the reverse serial order testing technique, see Phillips \& Christie, 1977a.) Each test pattern was laid on top of the card covering the corresponding pattem in the series, and the subject was required to place one white plastic square in each of the test matrices to make the pattern of white cells the same as in the original pattern. Each subject performed four such trials, after being given a single trial of practice, and the accuracy of each completion response was recorded by the experimenter.

At the end of the fourth trial and without prior warning, the test phase was repeated for all 16 patterns presented. The 16 incomplete test patterns were laid out once more in the order of original presentation so that the retention interval before this repeated test was approximately the same for all patterns. Subjects again placed a single square on each test pattern to make it the same as a previously presented pattern.

The order of presentation of patterns within the strings of four was balanced across subjects so that each pattern appeared equally of ten in each serial position.

\section{Results}

The percentage of correct completions for both immediate and final test is shown in Figure 4 as a function of serial presentation position. As each test pattern contained 10 blank cells, the probability of placing the missing cell correctly by chance is $10 \%$.

Each subject received a score out of 4 for each serial position at each test, representing the number of correct completion responses. Nonparametric statistical techniques were used to analyze these data. First, a Friedman two-way analysis of variance by ranks was performed on the initial test scores and yielded a highly significant effect of serial position $\left(\chi_{\mathrm{r}}^{2}=16.78, \mathrm{p}<.001\right)$. That this was due entirely to the superiority of the final position over the rest was confirmed in a repetition of the analysis of the scores for the first three serial positions alone, which showed no significant effect $\left(\chi_{\mathrm{r}}^{2}=.50\right)$.

A similar analysis performed on the repeated test scores failed to show a significant effect of serial position $\left(\chi_{\mathrm{r}}^{2}=2.14\right)$. The large positive recency effect evident at initial test is clearly absent on repeated test. The level of accuracy of Serial Positions 1-3 did not show any significant drop from initial to final test

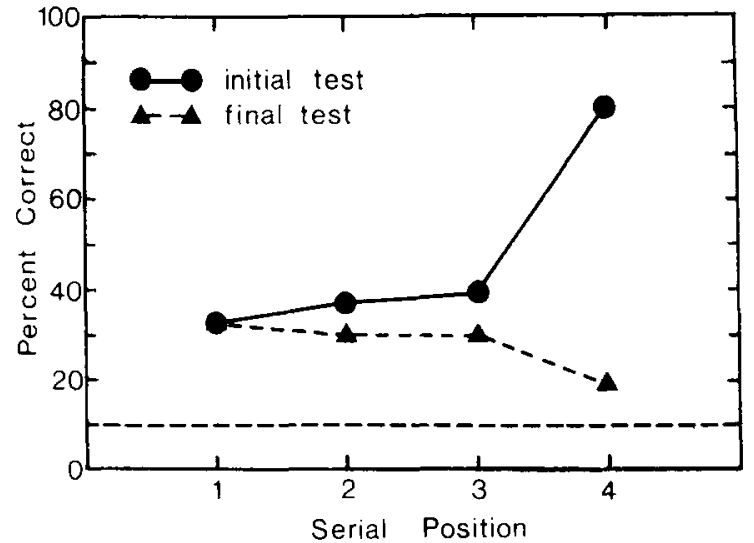

Figure 4. Mean percentage of correct completions in Experiment 5 as a function of serial position for both immediate and final test. Chance performance is $10 \%$.

(sign tests). However, there is some indication of a negative recency effect in the repeated test scores. This slight drop in performance for patterns that had been presented in Position 4 at initial test, as compared with those from other serial positions, achieves significance if the scores for Position 4 are compared with the average score for Positions 1.3 by sign test $(p=.019)$.

\section{Discussion}

The completion task is both simple and efficient. Experiment 5 required only $4 \mathrm{~h}$ total testing, yet produced a very clear difference between recency and longterm components. These components are very similar to those seen in the recognition and drawing tasks. The complete disappearance of the recency effect at final testing is further evidence that it reflects transitory aspects of processing, and not some form of LTM recency. Performance at final testing indicates that the longterm component is properly so described. The approximate equality of the long-term component at initial and final testing further indicates that the incomplete pattern is an effective retrieval cue. This completion technique is therefore particularly appropriate for testing over long series or over long intervals.

Further dissociation of recency and long-term components comes from the small negative recency effect that appears at final testing, and that also covers just one item. A possible reason for this negative recency effect is that at initial test less is learned about final than about nonfinal items. If so, the effect should disappear for items tested finally but not initially.

Experiment 5 differs from our previous serial position experiments in that the patterns were displayed successively at different places, rather than at the same place. It has been suggested that visual STM may be limited to one item only if successive items appear at the same place. Experiment 5 shows that the recency effect is still limited to one item, even when successive items are not in competition for the same locus within the scene. 


\section{GENERAL DISCUSSION}

The basic outcome of these experiments is that the distinction between STM and LTM previously observed in recognition tasks applies just as clearly to drawing and pattern completion tasks. The properties of these two components seem to be essentially the same in all three tasks. We suggest that this is because performance in all three tasks is based upon knowledge designed for general-purpose use and accessible to conscious voluntary processing.

The clear applicability of the distinction between STM and LTM to these visual tasks indicates that whatever underlies that distinction is not specific to language. There is more to STM than echo boxes and articulatory buffers. STM is not just a linguistic overflow system of only occasional use, as Weiskrantz (1970), for example, has suggested.

What, then, does underlie the distinction between LTM and STM? In everyday terms, this is easily answered. LTM is what we know, and STM is what we have in mind or are thinking about. This way of describing STM, however, is split between considering it as a kind of store and considering it as a kind of activity. Phillips and Christie (1977a, 1977b) have argued that these two aspects are not mutually exclusive, and that the storage aspects are supplementary to the processing functions. Experiment 2 provides further evidence for this view by showing that recency is removed by the recognition of recently learned patterns. The earlier experiments showed that recency is not removed by the recognition of familiar patterns such as digits. Thus interference with visualization does not depend upon whether subsequent representations are constructed but upon the kind of processing that their construction requires.

Emphasis upon processing functions may also help resolve the paradox of clear evidence for a visual analogy to verbal STM, but no evidence of short-term deterioration in performance. The flat functions relating performance to retention interval in Experiments 3 and 4 and the limitation of the recency effect to one item both show that visualized information is lost immediately or very shortly after attention is turned to other things. In terms of the classical conception of the STM-LTM distinction, it may seem reasonable to conclude that if there is no short-term deterioration in performance, then there is no functionally significant STM and, therefore, only LTM. For example, Frey and Adesman (1976), studying the recall of chess positions, found little evidence for deterioration in performance over filled intervals of 3 and $30 \mathrm{sec}$. They interpreted this as evidence against the proposal of Chase and Simon (1973) that a limited-capacity short-term visual memory is involved in chess skill. In our view, however, the absence of decay over filled intervals does not show that chess positions cannot be visualized. Our results do not indicate that visualized information remains in a distinct state after the subject has stopped visualizing it. But, nevertheless, they make clear that visualization occurs, and that it is distinct from long-term visual knowledge.

The discovery of temporary storage systems for holding phonemic or articulatory information may have been unfortunate in that it concentrated attention upon memory characteristics. Performance in the drawing, pattern completion, and recognition tasks supports the view that underlying much of the vast amount of evidence associated with STM and LTM is the more fundamental distinction between thought and knowledge.

\section{REFERENCE NOTE}

1. Baddeley, A. D., \& Hitch, G. Recency reexamined. Paper presented at the VI Symposium on Attention and Performance, Stockholm, 1975.

\section{REFERENCES}

Adamowicz, J. K., \& Hudson, B. R. Visual short-term memory, response delay, and age. Perceptual and Motor Skills, 1978, 46, 267-270.

BjoRk, R. A., \& Whitten, W. B. Recency-sensitive retrieval processes in long-term free recall. Cognitive Psychology, 1974, 6, 173-189.

Brown, J. Some tests of the decay theory of immediate memory. Quarterly Journal of Experimental Psychology, 1958, 10, 12-21.

Chase, W. G., \& Simon, H. A. Perception in chess. Cognitive Psychology, 1973, 4, 55-81.

Cohen, R. L., \& Granstrom, K. Reproduction and recognition in short-term visual memory. Quarterly Journal of Experimental Psychology, 1970, 22, 450-457.

Frey, P. W., \& Adesman, P. Recall memory for visually presented chess positions. Memory \& Cognition, 1976, 4, 541-547.

Kroll, N. E. A. Visual short-term memory. In D. Deutsch, J. A. Deutsch (Eds.), Short-term memory. New York: Academic Press, 1975.

Meudell, P. R. Effects of length of retention interval on proactive interference in short-term visual memory. Journal of Experimental Psychology: Human Learning and Memory, 1977, 3, 264-269.

Peterson, L. R., \& Peterson, M. J. Short-term retention of individual verbal items. Journal of Experimental Psychology, 1959, 58, 193-198.

Phillips, W. A., \& Baddeley, A. D. Reaction time and shortterm visual memory. Psychonomic Science, 1971, 22, 73-74.

Phillips, W. A., \& Christie, D. F. M. Components of visual memory. Quarterly Journal of Experimental Psychology, 1977, 29, 117-133. (a)

Phillips, W. A., \& Christie, D. F. M. Interference with visualization. Quarterly Journal of Experimental Psychology, 1977, 29, 637-650. (b)

Phillips, W. A., Hobis, S. B., \& Pratt, F. R. Intellectual realism in children's drawings of cubes. Cognition, 1978, 6, 15-33.

RASER, G. A. Recoding of semantic and acoustic information in short-term memory. Journal of Verbal Learning and Verbal Behavior, 1972, 11, 692-697.

RILEY, D. A. Memory for form. In L. Postman (Ed.), Psychology in the making. New York: Knopf, 1962.

Rock, I., \& Engelstein, P. A study of memory for visual form. American Journal of Psychology, 1959, 72, 221-229. 
Schnore, M. M., \& Partington, J. T. Immediate memory for visual patterns: Symmetry and amount of information. Psychonomic Science, 1967, 8, 421-422.

Shaffer, W. O., \& Shiffrin, R. M. Rehearsal and storage of visual information. Journal of Experimental Psychology, 1972, 92, 292-296.

Shulman, H. G. Encoding and retention of semantic and phonemic information in short-term memory. Journal of Verbal Learning and Verbal Behavior, 1970, 9, 499-508.

TAB AChNick, B., \& Brotsky, S. J. Free recall and complexity of pictorial stimuli. Memory \& Cognition, 1976, 4, 466-470.

TzenG, O. J. L. Positive recency effect in delayed free recall. Journal of Verbal Learning and Verbal Behavior, 1973, 12, 436-439.
Warrington, E. K., \& Weiskrantz, L. An analysis of shortterm and long-term memory defects in man. In J. A. Deutsch (Ed.), The physiological basis of memory. New York: Academic Press, 1973.

WEISKRANT2, L. A long-term view of short-term memory in psychology, in G. Horn \& R. A. Hinde (Eds.), Short-term changes in neural activity and behaviour. Cambridge, Eng: Cambridge University Press, 1970.

Yuille, J. C., \& Ternes, W. Attention and modality specific interference as determinants of short-term verbal and visual retention. Canadian Journal of Psychology, 1975, 29, 360-374.

(Received for publication July 20, 1978; revision accepted May $8,1979$. 